\title{
Regulation of melanoma malignancy by the RP11-705C15.3/miR-145-5p/NRAS/MAPK signaling axis
}

\author{
Xiang-jun Chen $\mathbb{D}^{1} \cdot$ Sha Liu ${ }^{1} \cdot$ Dong-mei Han ${ }^{1} \cdot$ De-zhi Han ${ }^{1} \cdot$ Wei-jing Sun ${ }^{1} \cdot$ Xiao-chun Zhao ${ }^{1}$
}

Received: 5 September 2020 / Revised: 4 November 2020 / Accepted: 23 November 2020 / Published online: 14 December 2020

(c) The Author(s), under exclusive licence to Springer Nature America, Inc. 2020. This article is published with open access

\begin{abstract}
Melanoma is a common lethal skin cancer. Dissecting molecular mechanisms driving the malignancy of melanoma may uncover potential therapeutic targets. We previously identified miR-145-5p as an important tumor-suppressive microRNA in melanoma. Here, we further investigated the roles of long non-coding RNAs (lncRNAs) in melanoma. We identified RP11705C15.3, a regulator of miR-145-5p, as an oncogenic IncRNA in melanoma. RP11-705C15.3 competitively bound miR$145-5 p$, relieved the repressive roles of miR-145-5p on its target NRAS, upregulated NRAS expression, and activated MAPK signaling. In vitro functional assays revealed that ectopic expression of RP11-705C15.3 promoted melanoma cell proliferation, inhibited apoptosis, and promoted migration and invasion. Silencing of RP11-705C15.3 repressed melanoma cell proliferation, induced apoptosis, and repressed migration and invasion. Notably, the roles of RP11-705C15.3 in melanoma cell proliferation, apoptosis, migration and invasion are reversed by miR-145-5p overexpression. In vivo functional assays revealed that RP11-705C15.3 promoted melanoma tumor growth and metastasis, which were also reversed by miR-145-5p overexpression. Furthermore, we investigated the expression of RP11-705C15.3 in clinical melanoma tissues and found that RP11-705C15.3 was increased in melanoma tissues. High expression of RP11-705C15.3 was positively correlated with thickness, ulceration, metastasis, and inferior overall survival. Taken together, our findings suggest RP11-705C15.3 as a novel oncogene in melanoma, and highlight that the RP11-705C15.3/miR-145-5p/NRAS/MAPK signaling axis may be potential therapeutic targets for melanoma.
\end{abstract}

\section{Introduction}

Melanoma, which arises from malignant transformation of melanocytes, is a common skin-related lethal cancer [1]. The incidence rate and mortality of melanoma have been still increased since the past 40 years [2]. According to global cancer statistics, there are approximate 288 thousand new melanoma cases and 61 thousand melanoma-caused deaths in 2018 [3]. Recent progressions in target therapies and checkpoint inhibitors have greatly improved the

These authors contributed equally: Xiang-jun Chen, Sha Liu

Supplementary information The online version of this article (https:// doi.org/10.1038/s41417-020-00274-5) contains supplementary material, which is available to authorized users.

Xiang-jun Chen cxj_253@163.com

1 Department of Burns and Plastic Surgery, The 969th Hospital of PLA, Hohhot, Inner Mongolia 010052, China outcome of melanomas [4]. However, the prognosis of melanomas, particular for those in late stages, is still inferior [5]. Hence, further elucidation of the detailed molecular mechanisms underlying melanoma progression would provide novel reasonable targets for melanoma therapy.

Recently, genomic and transcriptomic sequencings have identified approximate 25,000 protein-coding genes in human [6]. However, the genes encoding long non-coding RNAs (lncRNAs) are approximate 58,000, which are remarkably more abundant than that of protein-coding genes [6]. IncRNAs is another class of long transcripts with more than 200 nucleotides (nt) in length and limited protein-coding ability [7]. Current knowledge about IncRNAs shows that they are involved in the pathogenesis of various diseases, particular for cancers [8, 9]. In melanoma, several lncRNAs were recently reported to be associated with the initiation and progression of melanoma, such as SPRY4-IT1, Llme23, OVAAL, SRA, and LINC00520 [10-14]. In our previous reports, we also identified three oncogenic lncRNAs in melanoma: PVT1, ILF3-AS1, and MHENCR [15-17]. Due to the huge number of lncRNAs in 
human, other lncRNAs may also play important roles in melanoma.

lncRNAs exert their regulatory roles in pathophysiological processes via several different mechanisms, including epigenetic modification, protein interaction, and RNA interaction [18-20]. Among the RNA interaction, IncRNAs were frequently reported to bind microRNAs (miRNAs) [21, 22]. miRNA is another class of short regulatory RNA with 19-25nt in length [23]. Similar to IncRNAs, miRNAs are involved in various pathophysiological processes, including cancers $[24,25]$. They may exert oncogenic or tumor-suppressive roles in a variety of malignancies $[26,27]$. In our previous report, we identified miR-145-5p as an important tumor-suppressive miRNA in melanoma [28]. miR-145-5p was also found to be significantly downregulated in melanoma [28]. miRNAs exert their roles mainly via binding AGO2 to form RNA-induced silencing complex (RISC), which further bind target mRNAs and induce target mRNAs degradation and/or translation inhibition [29].

A particular class of lncRNAs could competitively bind miRNAs, relieve the repressive roles of miRNAs in their targets, and therefore relieve the biological functions of interacted miRNAs [21, 22]. These lncRNAs were also known as competing endogenous RNAs (ceRNAs) [21, 22]. Due to the important tumor-suppressive roles of miR-145$5 \mathrm{p}$ in melanoma, we searched the IncRNAs which function as ceRNAs to bind miR-145-5p in melanoma. In this study, we identified lncRNA RP11-705C15.3 (gene Name: AC010186.3, gene ID: ENSG00000257027) as a ceRNA to competitively bind miR-145-5p and therefore has oncogenic roles in melanoma. The expression, clinical reverence, roles, and mechanisms of action of RP11-705C15.3 in melanoma were detailedly investigated in this study.

\section{Materials and methods}

\section{Cell culture}

Human melanoma cell lines CHL-1 and SK-MEL-2 were obtained from Cell Resource Center, Chinese Academy of Sciences. CHL-1 and SK-MEL-2 cells were cultured in DMEM (Gibco) and MEM (Gibco) medium, respectively, supplemented with $10 \%$ fetal bovine serum (Gibco) at $37^{\circ} \mathrm{C}$ with $5 \% \mathrm{CO}_{2}$. Cell lines used in this study were authenticated by STR profiling and routinely tested as mycoplasma-free.

\section{RNA fluorescence in situ hybridization}

For in situ detection of RP11-705C15.3 in melanoma cells, the probes against RP11-705C15.3 were designed and produced by Advanced Cell Diagnostics (ACD). The hybridization and fluorescence detection were conducted using RNAscope Fluorescent Multiplex Detection Kit (ACD) following the manufacturer's manual. The subcellular localization of RP11-705C15.3 in CHL-1 cells was observed using the confocal laser scanning microscopy (Leica).

\section{Isolation of cytoplasmic and nuclear RNA}

Cytoplasmic and nuclear RNA of CHL-1 cells were isolated using the Cytoplasmic \& Nuclear RNA Purification Kit (Norgen) according to the manufacturer's instruction. The isolated RNA was detected by real-time PCR as described below.

\section{Real-time PCR}

The RNA isolated from tissues, total cells, cytoplasm, and nucleus, or RNA-RNA interaction enrichment was used to carry out reverse transcription to generate the first strand cDNA using the PrimeScript ${ }^{\mathrm{TM}}$ II 1st Strand cDNA Synthesis Kit (Takara, Dalian, China). Next, real-time PCR was carried out using TB Green Premix Ex ${ }^{\circ}{ }^{\mathrm{T}}{ }^{\mathrm{TM}}$ II (Takara) on StepOnePlus Real-Time PCR System (Thermo Fisher Scientific). The primer sequences were shown in Table 1. GAPDH was used as endogenous control for the quantification of RP11-705C15.3 and NRAS expression. For miRNAs quantification, real-time PCR was carried out using the TaqMan microRNA assays (Thermo Fisher Scientific) on StepOnePlus Real-Time PCR System following the manufacturer's manuals. The relative expression was calculated using $2^{-\Delta \Delta \mathrm{Ct}}$ method.

\section{RNA-RNA interaction detection}

To detect the RNAs bound to RP11-705C15.3, RP11$705 \mathrm{C} 15.3$ antisense biotinylated probes were designed and synthesized by LGC Biosearch Technology. The probe sequences were shown in Table 1. The RNA bound to RP11-705C15.3 was enriched using the probes and the EZMagna ChIRP RNA Interactome Kit (Millipore) following the manufacturer's manual. The enriched RNA was detected by real-time PCR as described above. In addition, in vitro transcribed biotinylated RP11-705C15.3 was used to enrich the RNAs which could bind to RP11-705C15.3. RP11-705C15.3 full-length sequences were PCR-amplified and further cloned into the Kpn I and Hind III sites of pSPT19 vector (Roche) to generate pSPT19-RP11705C15.3. The primers sequences were shown in Table 1. The PCR products were miR-145-5p binding sites mutated RP11-705C15.3 was synthesized by GenScript (Nanjing, China) and cloned into the Kpn I and Hind III sites of 
Table 1 The primers and probes sequences.

\begin{tabular}{|c|c|}
\hline Gene & Sequence $\left(5^{\prime} \rightarrow 3^{\prime}\right)$ \\
\hline \multicolumn{2}{|l|}{ Real-time PCR primers } \\
\hline RP11-705C15.3 forward & 5'-CAGGGGTGGTGGATCACA-3' \\
\hline RP11-705C15.3 reverse & 5'-CAACTCCAAGCCCGCTTAA-3' \\
\hline NRAS forward & 5'-GAAATACGCCAGTACCGAATG-3' \\
\hline NRAS reverse & 5'-TTCTCCTCCAGGGAAGTCAG-3' \\
\hline GAPDH forward & 5'-GTCGGAGTCAACGGATTTG-3' \\
\hline GAPDH reverse & 5'-TGGGTGGAATCATATTGGAA-3' \\
\hline \multirow{8}{*}{$\begin{array}{l}\mathrm{RP} 11-705 \mathrm{C} 15.3 \\
\text { antisense probes }\end{array}$} & 5'-ATGACATTTCAAGCCAAACC-3' \\
\hline & 5'-AAACATGCTGGCAAACAGCA-3' \\
\hline & 5'-GTGAGTCTTGAAAGTCCCAA-3' \\
\hline & 5'-TTTCAGATGGAGTTGTTTCC-3' \\
\hline & 5'-ACTTCTTGCTGATTAGGGAC-3' \\
\hline & 5'-TCAGTCTGGAGCAGTTACAA-3' \\
\hline & 5'-CACTCTGTAAATGAGGTAGC-3' \\
\hline & 5'-AAGCCCCCATAAAGCATGTG-3' \\
\hline \multicolumn{2}{|l|}{ Vectors construction primers } \\
\hline $\begin{array}{l}\text { pSPT19-RP11-705C15.3 } \\
\text { forward }\end{array}$ & 5'-GGGGTACCGGTTTGGCTTGAAATGTCATA-3' \\
\hline $\begin{array}{l}\text { pSPT19-RP11-705C15.3 } \\
\text { reverse }\end{array}$ & 5'-CCCAAGCTTTGTTACCATAAAAGTTGAACC-3' \\
\hline $\begin{array}{l}\text { pmirGLO-RP11-705C } 15.3 \\
\text { forward }\end{array}$ & 5'-CTAGCTAGCTTTGGCTAAGCATTTGACTC-3' \\
\hline $\begin{array}{l}\text { pmirGLO-RP11-705C } 15.3 \\
\text { reverse }\end{array}$ & 5'-CCCTCGAGACAGCACATTCCTCCTC-3' \\
\hline pmirGLO-NRAS forward & 5'-CTAGCTAGCCCCAGGAGAAAGATGAAAC-3' \\
\hline pmirGLO-NRAS reverse & 5'-CGCTCGAGATGACTAAGCCAAGAACTTC-3' \\
\hline \multicolumn{2}{|c|}{ cDNA oligonucleotides sequences for RP11-705C15.3 shRNAs } \\
\hline LV-shRNA-1 forward & 5'-GATCCGCTGTTTGCCAGCATGTTTGATTCAAGAGATCAAACATGCTGGCAAACAGCTTTTTTG-3' \\
\hline LV-shRNA-1 reverse & 5'-AATTCAAAAAAGCTGTTTGCCAGCATGTTTGATCTCTTGAATCAAACATGCTGGCAAACAGCG-3' \\
\hline LV-shRNA-2 forward & 5'-GATCCGGAAACAACTCCATCTGAAATTTCAAGAGAATTTCAGATGGAGTTGTTTCCTTTTTTG-3' \\
\hline LV-shRNA-2 reverse & 5'-AATTCAAAAAAGGAAACAACTCCATCTGAAATTCTCTTGAAATTTCAGATGGAGTTGTTTCCG-3' \\
\hline LV-shNC forward & 5'-GATCCGTTCTCCGAACGTGTCACGTTTCAAGAGAACGTGACACGTTCGGAGAACTTTTTTG-3' \\
\hline LV-shNC reverse & 5'-AATTCAAAAAAGTTCTCCGAACGTGTCACGTTCTCTTGAAACGTGACACGTTCGGAGAACG-3' \\
\hline
\end{tabular}

pSPT19 vector (Roche) to generate pSPT19-RP11705C15.3-mut. Wild type and miR-145-5p binding sites mutated RP11-705C15.3 were in vitro transcribed from pSPT19-RP11-705C15.3 and pSPT19-RP11-705C15.3mut, respectively, and concurrently being biotinylated with the Biotin RNA Labeling Mix (Roche) and Sp6 RNA polymerase (Roche). After purification, $3 \mu \mathrm{g}$ of wild type or miR-145-5p binding sites mutated RP11-705C15.3 were incubated with $1 \mathrm{mg}$ of whole-cell lysates from CHL-1 cells at $25^{\circ} \mathrm{C}$ for $1 \mathrm{~h}$. Next, streptavidin agarose beads (Thermo Fisher Scientific) were added to enrich biotinylated wild type or miR-145-5p binding sites mutated RP11-705C15.3 and its interacted RNAs. The enriched RNA was detected by real-time PCR as described above.

\section{RNA Immunoprecipitation (RIP) assays}

miR-145-5p and miR-1-3p mimics and negative control (NC) were purchased from GenePharma (Shanghai, China) and transfected into CHL-1 cells using Lipofectamine 3000 (Thermo Fisher Scientific) according to the manufacturer's manual. Forty-eight hours after transfection, RIP assays were performed using the Magna RIPTM RNA-Binding Protein Immunoprecipitation Kit (Millipore) and 
anti-AGO2 antibody (Cat\# 03-110, $5 \mu \mathrm{L}$, Millipore) following the manufacturer's manual. The enriched RNA was detected by real-time PCR as described above.

\section{Dual luciferase reporter assays}

RP11-705C15.3 sequences containing miR-145-5p binding sites were PCR-amplified and further cloned into the Nhe I and Xho I sites of pmirGLO (Promega) to generate pmirGLO-RP11-705C15.3. The primers sequences were shown in Table 1. The corresponding miR-145-5p binding sites mutated pmirGLO-RP11-705C15.3 (pmirGLO-RP11705C15.3-mut) was constructed as above except using pSPT19-RP11-705C15.3-mut as the template. NRAS 3'UTR containing miR-145-5p binding sites was PCRamplified and further cloned into the Nhe I and Xho I sites of pmirGLO to generate pmirGLO-NRAS. The primers sequences were shown in Table 1 . The corresponding miR145-5p binding sites mutated pmirGLO-NRAS (pmirGLONRAS-mut) was synthesized by GenScript (Nanjing, China). pmirGLO, pmirGLO-RP11-705C15.3, or pmirGLO-RP11-705C15.3-mut was co-transfected with miR-145-5p or miR-1-3p mimics or miR-NC into CHL-1 cells using Lipofectamine 3000. Forty-eight hours after transfection, the luciferase activities were detected using the Dual-Luciferase Reporter Assay System (Promega). pmirGLO, pmirGLO-NRAS, or pmirGLO-NRAS-mut was transfected into CHL-1 cells overexpressing or silencing RP11-705C15.3 using Lipofectamine 3000. Forty-eight hours after transfection, the luciferase activities were detected using the Dual-Luciferase Reporter Assay System.

\section{The construction of melanoma cells stably overexpressing or silencing RP11-705C15.3}

To construct RP11-705C15.3 stably overexpressed melanoma cells, RP11-705C15.3 overexpressing lentivirus (LV11/CMV/Neo) were purchased from GenePharma (Shanghai, China) and infected into CHL-1 and SK-MEL-2 cells. Then, the cells were treated with neomycin for four weeks to select RP11-705C15.3 overexpressed cells. Two pairs of cDNA oligonucleotides suppressing RP11$705 \mathrm{C} 15.3$ expression were designed and synthesized by GenePharma. After annealing, double-strand oligonucleotides were inserted into the lentiviral vector pLV6/EF-1a/ Puro to produce shRNA lentivirus suppressing RP11$705 \mathrm{C} 15.3$ expression. Then, the lentivirus was infected into CHL-1 and SK-MEL-2 cells. The cells were treated with puromycin for four weeks to select RP11$705 \mathrm{C} 15.3$ silenced cells. The cDNA oligonucleotides sequences were shown in Table 1. To construct melanoma cells overexpressing RP11-705C15.3 and miR-145-5p, RP11-705C15.3 overexpressed CHL-1 cells were infected with miR-145-5p overexpressing lentivirus (Genechem Co. Ltd., Shanghai, China) and treated with neomycin and puromycin for 4 weeks to select RP11-705C15.3 and miR$145-5 p$ overexpressed cells.

\section{Western blot}

Western blot was performed as we previously described [17]. Primary antibodies used were as follows: for NRAS, ab154291, 1:1000, Abcam; for GAPDH, ab8245, 1:5000, Abcam; for phospho-MEK1/2, \#9154, 1:1000, Cell Signaling Technology; for MEK1/2, \#8727, 1:1000, Cell Signaling Technology; for phospho-ERK1/2, \#4370, 1:2000, Cell Signaling Technology; for ERK1/2, \#4695, 1:1000, Cell Signaling Technology.

\section{Cell viability, proliferation, apoptosis, migration, and invasion assays}

Cell viability was measured by the Glo cell viability assay as we previously described [16]. Briefly, 3000 indicated melanoma cells were seeded into 96-well plates per well. At the indicated time, the luminescence values were measured using the Cell Titer-Glo Luminescent Cell Viability Assay (Promega) to indicate cell viability. Cell proliferation was measured using the ethynyl deoxyuridine (EdU) incorporation assay as we previously described [16]. EdU incorporation assay was carried out using the EdU Kit (Roche) following the manufacturer's manual. Cell apoptosis was measured using the Annexin V-FITC apoptosis detection kit (BD Pharmingen) as we previously described [28]. Cell migration was measured using the transwell migration assay as we previously described [16]. Cell invasion was measured using the transwell invasion assay as we previously described [16].

\section{Animal studies}

Five-week old male athymic BALB/c nude mice were purchased from Chinese Academy of Sciences and maintained in pathogen-free condition. A total of $2.0 \times 10^{6}$ indicated melanoma cells were subcutaneously inoculated into the flanks of nude mice. Subcutaneous tumor volumes were measured using caliper every 7 days and calculated using the formula $\mathrm{V}=0.5 \times \mathrm{LW}^{2}$ (L, tumor length; W, tumor width). At the 28th day after inoculation, subcutaneous tumors were resected and weighed. The tumors were further used to perform immunohistochemistry (IHC) staining with the antibody against Ki67 (ab15580, $1 \mu \mathrm{g} / \mathrm{mL}$, Abcam). The subcutaneous tumors were also used to carry out TdT-mediated dUTP Nick-End Labeling (TUNEL) staining with the One Step TUNEL Apoptosis Assay Kit (Beyotime, Shanghai, China). To detect melanoma liver 
metastasis in vivo, $2.0 \times 10^{6}$ indicated melanoma cells were intrasplenically inoculated into nude mice to construct liver metastasis model. At the 28th day after inoculation, the mice were sacrificed and the livers were resected and used to perform hematoxylin-eosin (H\&E) staining. To detect melanoma lung metastasis in vivo, $2.0 \times 10^{6}$ indicated melanoma cells were inoculated into tail vein of nude mice to construct lung metastasis model. At the 28th day after inoculation, the mice were sacrificed and the lungs were resected and used to perform H\&E staining. Animal studies were performed following the protocols approved by the Review Board of the 969th Hospital of PLA (Hohhot, Inner Mongolia, China). The experiments were not randomized. No statistical method was used to determine sample size. The experimenters recording tumor growth and metastasis were blinded to mice allocation.

\section{Human tissue samples}

Sixty-eight cutaneous malignant melanoma tissues and thirty-six age and gender-matched skin tissues with melanocytic nevus were acquired from patients who received surgery at the 969th Hospital of PLA (Hohhot, Inner Mongolia, China) with written informed consent. All human tissues were confirmed by histopathological examination. The Review Board of the 969th Hospital of PLA reviewed and approved the use of human tissues.

\section{Statistical analysis}

GraphPad Prism Software was used to carry out all statistical analyses. For comparisons, one-way ANOVA followed by Dunnett's multiple comparisons test, two-tailed unpaired $t$ test, one-way ANOVA followed by Tukey's multiple comparisons test, Kruskal-Wallis test followed by Dunn's multiple comparisons test, Mann-Whitney test, or log-rank test were performed as indicated in figure legends. $p<0.05$ was considered as statistically significant.

\section{Results}

\section{RP11-705C15.3 was identified to directly bind miR- 145-5p}

The IncRNAs which could bind miR-145-5p was searched using the online in silico tool the Encyclopedia of RNA Interactomes (ENCORI) (http://starbase.sysu.edu.cn/) [30]. We focused on IncRNA RP11-705C15.3, which has two predicted miR-145-5p binding sites locating at 713-719nt and 1043-1049nt of RP11-705C15.3 (Fig. 1A). Furthermore, The Cancer Genome Atlas (TCGA) Skin Cutaneous Melanoma (SKCM) dataset showed that RP11-705C15.3 was upregulated in melanoma, analyzed by the Gene Expression Profiling Interactive Analysis (GEPIA) (http:// gepia.cancer-pku.cn/) (Supplementary Fig. 1a). Confocal RNA FISH indicated that RP11-705C15.3 was mainly located in the cytoplasm (Fig. 1B). Cytoplasmic or nuclear RNA purification followed by real-time PCR also indicated the cytoplasmic localization of RP11-705C15.3 (Fig. 1C), which support the potential interaction between RP11$705 \mathrm{C} 15.3$ and miR-145-5p. To investigate whether RP11$705 \mathrm{C} 15.3$ could bind miR-145-5p, we retrieved RP11$705 \mathrm{C} 15.3$ and its interacted RNAs using RP11-705C15.3 antisense probes. The results indicated that RP11-705C15.3 was successfully enriched using the antisense probes, and also miR-145-5p but not miR-1-3p was enriched in RP11$705 \mathrm{C} 15.3$ antisense probes group (Fig. 1D). Moreover, we also used in vitro transcribed biotin-labeled wild-type or miR-145-5p binding sites mutated RP11-705C15.3 to enrich its interacted RNAs. The results indicated that miR145-5p but not miR-1-3p was significantly enriched in wildtype RP11-705C15.3 group, which was abolished by the mutation of miR-145-5p binding sites in RP11-705C15.3 (Fig. 1E). miRNAs were known to bind $\mathrm{AGO} 2$, and then the miRNAs-AGO2 complex binds to their targets [29]. Thus, we performed anti-AGO2 RIP in melanoma cells after transient transfection of miR-145-5p mimics. As shown in Fig. 1F, miR-145-5p overexpression significantly increased the binding between RP11-705C15.3 and AGO2, further supporting the interaction between miR-145-5p and RP11-705C15.3. Luciferase reporter containing wild-type RP11-705C15.3 or miR-145-5p binding sites mutated RP11-705C15.3 was constructed. Dual luciferase reporter assays indicated that overexpression of miR-145-5p significantly reduced the luciferase activities of the reporter containing wild-type RP11-705C15.3, which was abolished by the mutation of miR-145-5p binding sites on RP11705C15.3 (Fig. 1G-I). The coding potential of RP11$705 \mathrm{C} 15.3$ was calculated by two in silico tools, namely the Coding Potential Assessment Tool (CPAT) (http://lilab. research.bcm.edu/cpat/index.php) and the Coding Potential Calculator (CPC) (http://cpc2.cbi.pku.edu.cn/). CPC and CPAT scores of RP11-705C15.3 were equally low as wellknown lncRNA HOTAIR (Supplementary Fig. 1b, c), which indicated the noncoding nature of RP11-705C15.3. Thus, these findings suggested that noncoding RNA RP11$705 \mathrm{C} 15.3$ physically bound miR-145-5p.

\section{RP11-705C15.3 activated NRAS/MAPK signaling via competitively binding miR-145-5p}

In our previous report, we have documented that miR-145$5 p$ represses MAPK signaling via directly targeting NRAS [28]. Thus, we further investigated whether RP11-705C15.3 regulates NRAS/MAPK signaling via competitively binding 

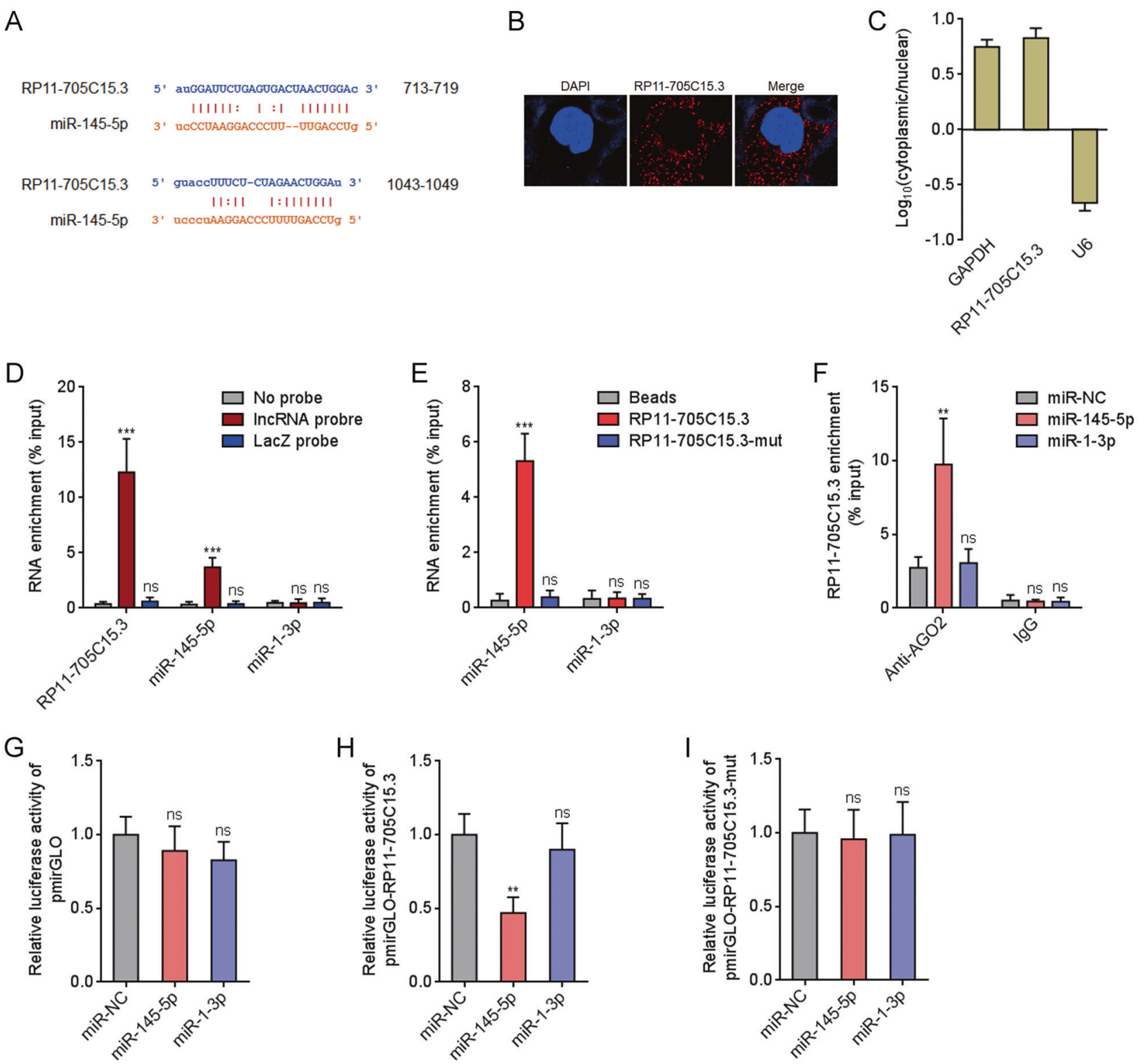

Fig. 1 RP11-705C15.3 physically bound to miR-145-5p. A The two predicted miR-145-5p binding sites on RP11-705C15.3. B Confocal RNA FISH images showed cytoplasmic localization of RP11705C15.3 in CHL-1 cells. C The levels of RP11-705C15.3 in purified cytoplasmic or nuclear RNAs derived from CHL-1 cells were detected by real-time PCR. GAPDH and U6 serve as cytoplasmic and nuclear controls, respectively. D RP11-705C15.3 antisense probes were used to enrich RP11-705C15.3 and its interacted RNAs in CHL-1 cells, followed by real-time PCR detection. E In vitro transcribed biotin-labeled wild-type or miR-145-5p binding sites mutated RP11$705 \mathrm{C} 15.3$ were used to enrich the RNAs bound to wild-type or mutant RP11-705C15.3 in CHL-1 cells, followed by real-time PCR detection. F Anti-AGO2 RIP assays were performed in CHL-1 cells after

miR-145-5p. NRAS 3'UTR containing wild-type or mutant miR-145-5p binding sites were inserted into luciferase reporter pmirGLO. Dual luciferase reporter assays indicated that overexpression of RP11-705C15.3 significantly increased the luciferase activities of the reporter containing transient transfection of miR-145-5p or miR-1-3p mimics, followed by real-time PCR detection to measure AGO2-bound RP11-705C15.3. G-I After transient co-transfection of empty luciferase reporter (pmirGLO) (G), luciferase reporter containing wild-type RP11705C15.3 (pmirGLO-RP11-705C15.3) (h), or luciferase reporter containing miR-145-5p binding sites mutated RP11-705C15.3 (pmirGLO-RP11-705C15.3-mut) (I) and miR-145-5p or miR-1-3p mimics into CHL-1 cells, the luciferase activities were measured. Results are shown as the relative ratio of firefly luciferase activity to renilla luciferase activity. For $\mathbf{C}-\mathbf{I}$, data are presented as mean \pm SD based on three independent experiments. $* * p<0.01, * * * p<0.001$, ns, not significant, by one-way ANOVA followed by Dunnett's multiple comparisons test.

wild-type NRAS, but not mutant NRAS (Fig. 2A-C). Furthermore, the increased luciferase activities were abolished by the mutation of miR-145-5p binding sites on RP11-705C15.3 (Fig. 2B), which suggested that the effects of RP11-705C15.3 on NRAS were dependent on the 
A
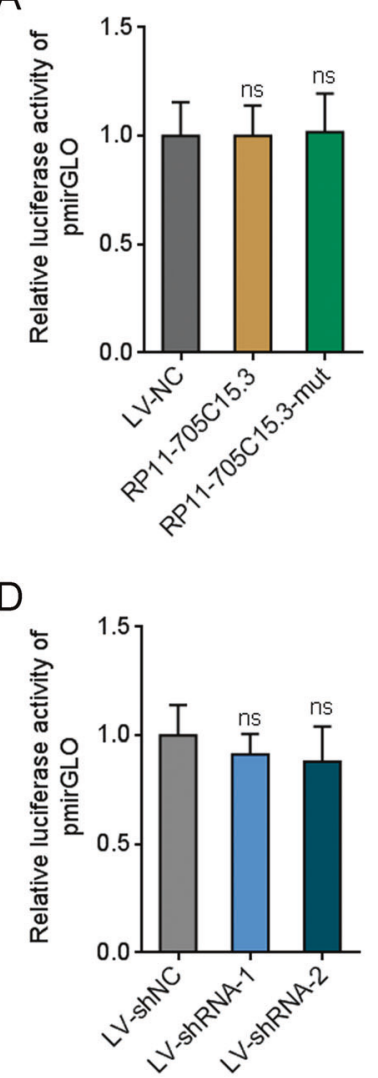

I

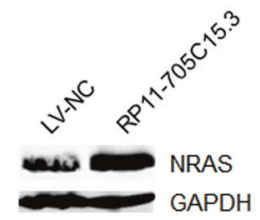

B

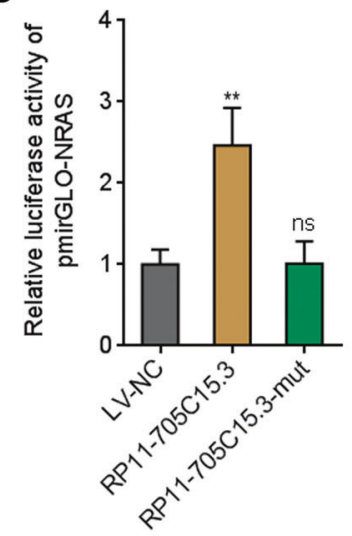

E

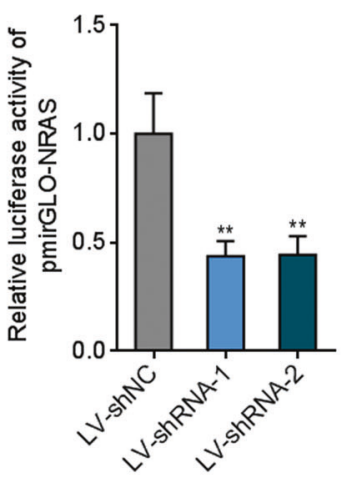

C

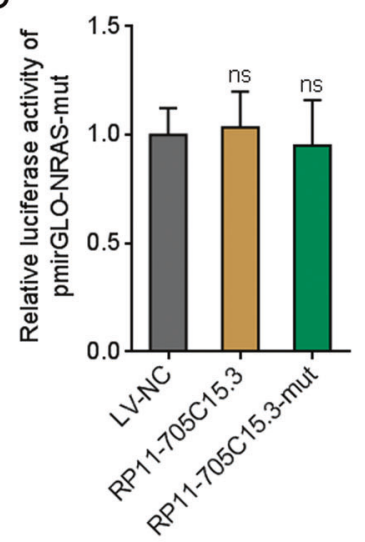

$\mathrm{F}$

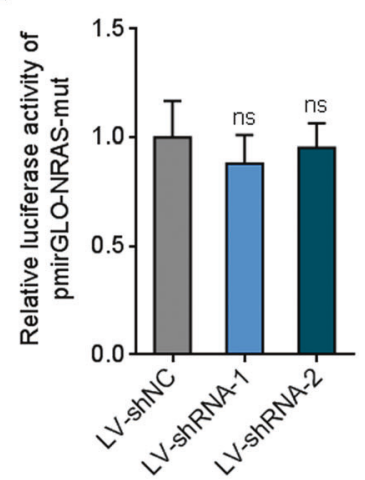

G

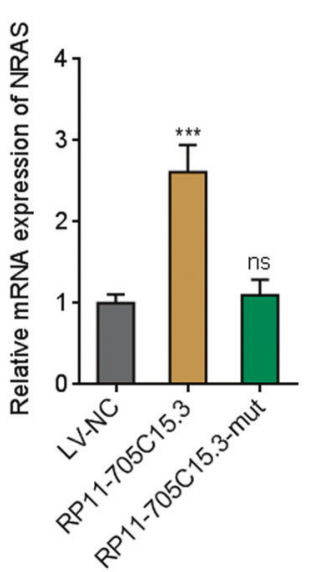

$\mathrm{H}$

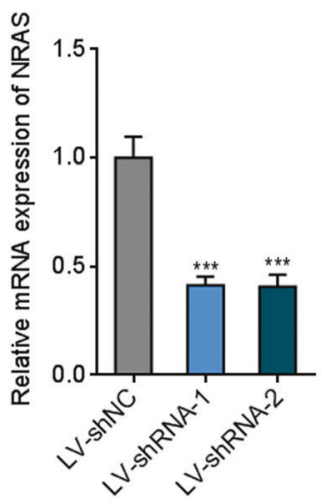

Fig. 2 RP11-705C15.3 activated NRAS/MAPK signaling. A-C After transient transfection of empty luciferase reporter (pmirGLO) (A), luciferase reporter containing wild-type NRAS 3'UTR (pmirGLO-NRAS) (B), or luciferase reporter containing miR-145-5p binding sites mutated NRAS 3'UTR (pmirGLO-NRAS-mut) (C) into CHL-1 cells overexpressing wild-type or miR-145-5p binding sites mutated RP11-705C15.3, the luciferase activities were measured. Results are shown as the relative ratio of firefly luciferase activity to renilla luciferase activity. D-F After transient transfection of pmirGLO (D), pmirGLO-NRAS (E), or pmirGLO-NRAS-mut (F) into CHL-1 cells silencing RP11-705C15.3 or control, the luciferase activities were measured. Results are shown as the relative ratio of firefly luciferase activity to renilla luciferase activity. G NRAS mRNA expression levels in CHL-1 cells overexpressing wild-type or miR-145-5p binding

regulation of miR-145-5p. Conversely, dual luciferase reporter assays revealed that RP11-705C15.3 silencing decreased the luciferase activities of the reporter containing wild-type NRAS, but not mutant NRAS (Fig. 2D-F). Overexpression of RP11-705C15.3 remarkably upregulated

$\mathrm{K}$

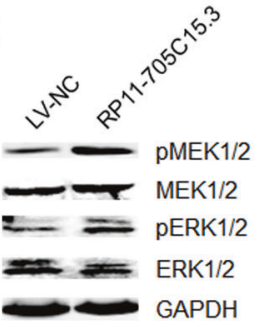

$\mathrm{L}$

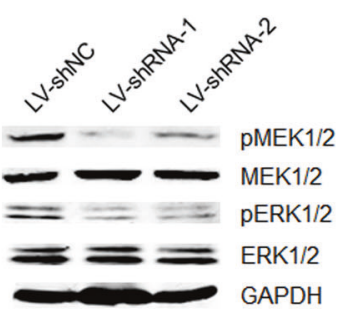

sites mutated RP11-705C15.3 were measured by real-time PCR. H NRAS mRNA expression levels in CHL-1 cells silencing RP11$705 \mathrm{C} 15.3$ or control were measured by real-time PCR. I NRAS protein levels in CHL-1 cells overexpressing RP11-705C15.3 or control were measured by western blot. J NRAS protein levels in CHL-1 cells silencing RP11-705C15.3 or control were measured by western blot. $\mathbf{K}$ Phosphorylation levels of MEK1/2 and ERK1/2 in CHL-1 cells overexpressing RP11-705C15.3 or control were measured by western blot. L Phosphorylation levels of MEK1/2 and ERK1/2 in CHL-1 cells silencing RP11-705C15.3 or control were measured by western blot. Data are presented as mean \pm SD based on three independent experiments. $* * p<0.01$, ***p $p<0.001$, ns, not significant, by one-way ANOVA followed by Dunnett's multiple comparisons test.

the mRNA levels of NRAS, which was abolished by the mutation of miR-145-5p binding sites on RP11-705C15.3 (Fig. 2G). Conversely, RP11-705C15.3 silencing remarkably downregulated the mRNA levels of NRAS (Fig. 2H). Consistently, NRAS protein levels were increased in 
melanoma cells overexpressing RP11-705C15.3 and decreased in melanoma cells silencing RP11-705C15.3 (Fig. 2I, J). Next, the effects of RP11-705C15.3 on MAPK signaling were investigated. The phosphorylation levels of MEK1/2 and ERK1/2 were increased in melanoma cells overexpressing RP11-705C15.3 and decreased in melanoma cells silencing RP11-705C15.3 (Fig. 2K, L). Collectively, these findings suggested that RP11-705C15.3 activated NRAS/MAPK signaling in a miR-145-5p dependent manner in melanoma.

\section{RP11-705C15.3 promoted melanoma cell proliferation, repressed apoptosis, and promoted migration and invasion in vitro}

Due to the binding of miR-145-5p and activation of NRAS/ MAPK signaling by RP11-705C15.3, we next investigated the potential biological roles of RP11-705C15.3 in melanoma. RP11-705C15.3 stably overexpressed and control CHL-1 and SK-MEL-2 cells were constructed via RP11$705 \mathrm{C} 15.3$ overexpression lentivirus-mediated infection (Fig. 3A, B). Glo cell viability assays revealed that both CHL-1 and SK-MEL-2 cells overexpressing RP11$705 \mathrm{C} 15.3$ had increased cell viabilities compared with control cells (Fig. 3C, D). EdU incorporation assays revealed that both CHL-1 and SK-MEL-2 cells overexpressing RP11-705C15.3 had quicker cell proliferation rates compared with control cells (Fig. 3E). FITC-Annexin V/PI staining followed by flow cytometric analysis revealed that both CHL-1 and SK-MEL-2 cells overexpressing RP11-705C15.3 had less apoptotic cells compared with control cells (Fig. 3F). Transwell migration assays revealed that both CHL-1 and SK-MEL-2 cells overexpressing RP11-705C15.3 had more migrated cells compared with control cells (Fig. 3G). Transwell invasion assays revealed that both CHL-1 and SK-MEL-2 cells overexpressing RP11-705C15.3 had more invasive cells compared with control cells (Fig. 3H).

RP11-705C15.3 stably silenced and control CHL-1 and SK-MEL-2 cells were further constructed via two different independent RP11-705C15.3 specific shRNAs lentivirusmediated infection (Fig. 4A, B). Glo cell viability assays revealed that CHL-1 and SK-MEL-2 cells silencing RP11$705 \mathrm{C} 15.3$ had reduced cell viabilities compared with control cells (Fig. 4C, D). EdU incorporation assays further revealed that CHL-1 and SK-MEL-2 cells silencing RP11$705 \mathrm{C} 15.3$ had slower cell proliferation rates compared with control cells (Fig. 4E). FITC-Annexin V/PI staining followed by flow cytometric analysis revealed that CHL-1 and SK-MEL-2 cells silencing RP11-705C15.3 had more apoptotic cells compared with control cells (Fig. 4F). Transwell migration assays revealed that CHL-1 and SKMEL-2 cells silencing RP11-705C15.3 had less migrated cells compared with control cells (Fig. 4G). Transwell invasion assays revealed that CHL-1 and SK-MEL-2 cells silencing RP11-705C15.3 had less invasive cells compared with control cells (Fig. 4H). Therefore, these findings demonstrated that RP11-705C15.3 promoted melanoma cell proliferation, repressed apoptosis, and promoted migration and invasion in vitro.

\section{miR-145-5p reversed the roles of RP11-705C15.3 in melanoma cell proliferation, apoptosis, migration, and invasion}

To elucidate whether the oncogenic roles of RP11$705 \mathrm{C} 15.3$ in melanoma are dependent on the regulation of miR-145-5p, we stably overexpressed miR-145-5p in CHL1 cells overexpressing RP11-705C15.3 (Fig. 5A). Glo cell viability assays revealed that overexpression of miR-145-5p reversed the increased cell viability caused by RP11$705 \mathrm{C} 15.3$ (Fig. 5B). EdU incorporation assays revealed that overexpression of miR-145-5p reversed the accelerated cell proliferation caused by RP11-705C15.3 (Fig. 5C). FITCAnnexin V/PI staining followed by flow cytometric analysis revealed that overexpression of miR-145-5p reversed the reduced cell proliferation caused by RP11-705C15.3 (Fig. 5D). Transwell migration assays revealed that overexpression of miR-145-5p reversed the increased cell migration caused by RP11-705C15.3 (Fig. 5E). Transwell invasion assays revealed that overexpression of miR-145-5p reversed the increased cell invasion caused by RP11$705 \mathrm{C} 15.3$ (Fig. 5F). Thus, these finding suggested that the oncogenic roles of RP11-705C15.3 in melanoma cell proliferation, apoptosis, migration, and invasion are dependent on the regulation of miR-145-5p.

\section{RP11-705C15.3 promoted melanoma growth and metastasis in vivo via regulating miR-145-5p}

To further investigate the biological roles of RP11705C15.3/miR-145-5p modulatory axis in melanoma, RP11-705C15.3 overexpressed CHL-1 cells with or without miR-145-5p overexpression were subcutaneously injected into nude mice. Tumor volumes were measured every 7 days and the tumors were excised and weighed at the 28th day after injection. As shown in Fig. 6A, B, the tumors formed by RP11-705C15.3 overexpressed CHL-1 cells had quicker growth rates and formed larger tumors compared with the tumors formed by control cells. The growthpromoting roles of RP11-705C15.3 were abolished by concurrent miR-145-5p overexpression (Fig. 6A, B). Proliferation marker $\mathrm{Ki67} \mathrm{IHC}$ staining revealed that the tumors formed by RP11-705C15.3 overexpressed CHL-1 cells had more Ki67 positive cells compared with the tumors formed by control cells (Fig. 6C). The increased 

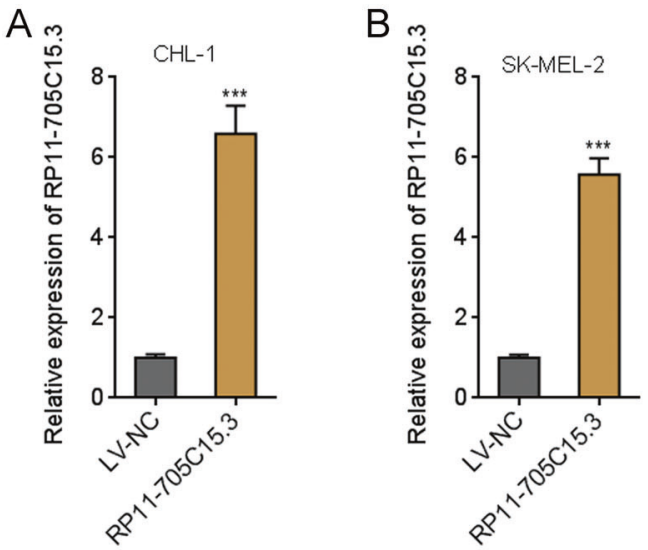

C

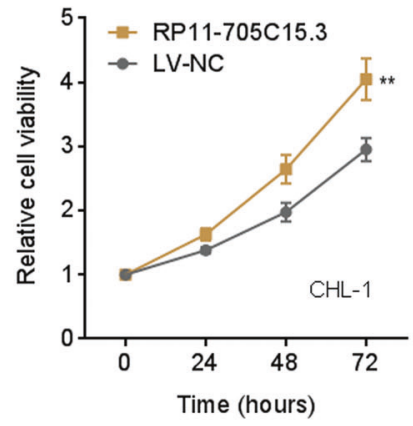

D

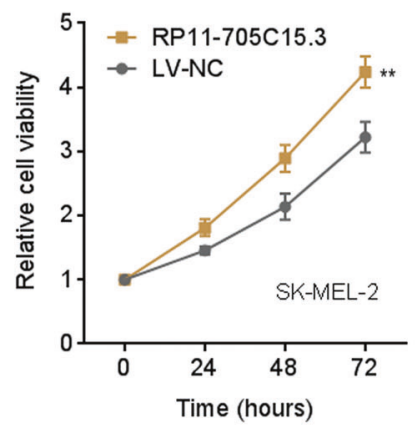

E

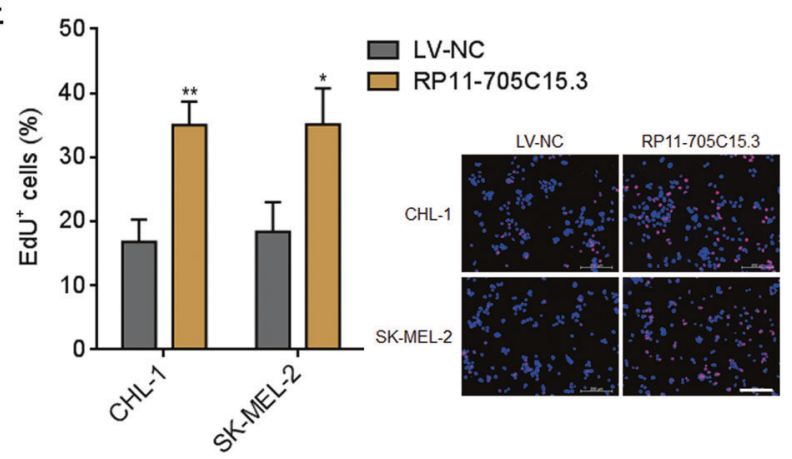

G

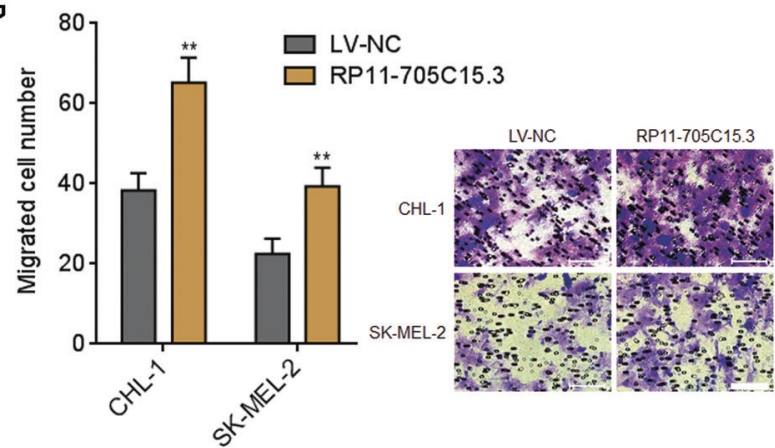

Fig. 3 RP11-705C15.3 promoted melanoma cell proliferation, inhibited apoptosis, and promoted migration and invasion. A RP11-705C15.3 expression in CHL-1 cells overexpressing RP11$705 \mathrm{C} 15.3$ or control was measured by real-time PCR. B RP11-705C15.3 expression in SK-MEL-2 cells overexpressing RP11$705 \mathrm{C} 15.3$ or control was measured by real-time PCR. C Cell viabilities of CHL-1 cells overexpressing RP11-705C15.3 or control were determined by the Glo cell viability assay. D Cell viabilities of SKMEL-2 cells overexpressing RP11-705C15.3 or control were determined by the Glo cell viability assay. E Cell proliferation of CHL-1 and SK-MEL-2 cells overexpressing RP11-705C15.3 or control were determined by the EdU incorporation assay. The red color indicates

number of Ki67 positive cells was abolished by concurrent miR-145-5p overexpression (Fig. 6C). TUNEL staining revealed that the tumors formed by RP11-705C15.3 overexpressed CHL-1 cells had less apoptotic cells compared
$\mathrm{F}$
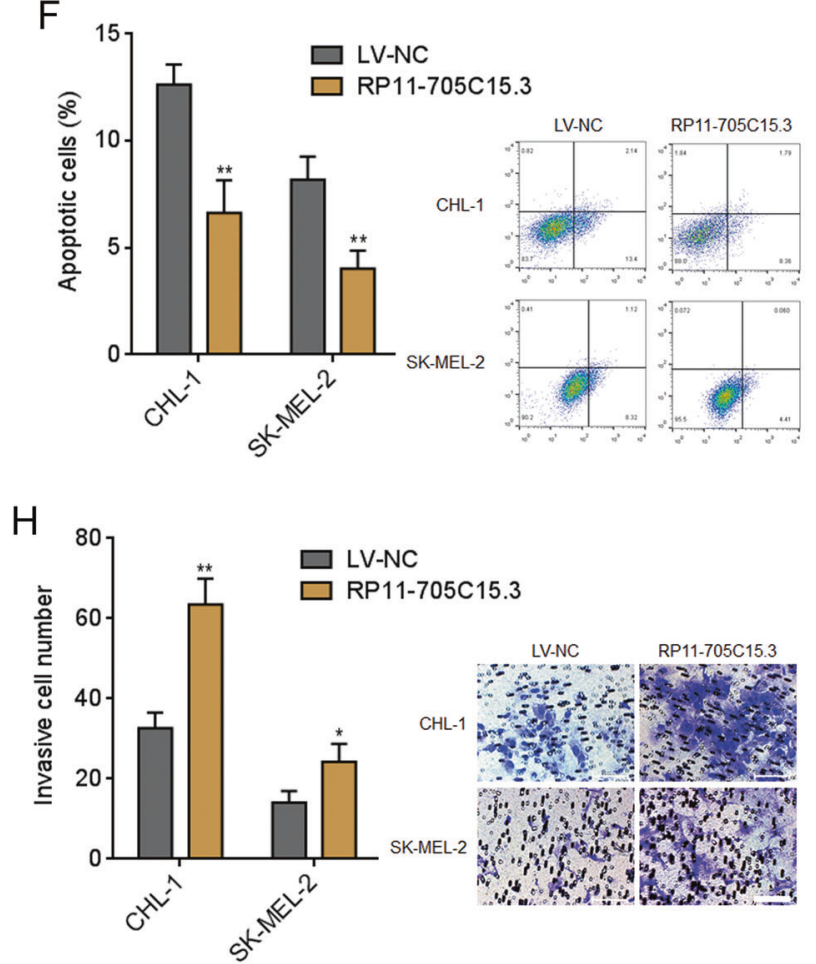

EdU-positive nuclei. Scale bars, $200 \mu \mathrm{m}$. F Cell apoptosis of CHL-1 and SK-MEL-2 cells overexpressing RP11-705C15.3 or control were determined by FITC-Annexin V/PI staining followed by flow cytometric analysis. G Cell migration of CHL-1 and SK-MEL-2 cells overexpressing RP11-705C15.3 or control were determined by the transwell migration assay. Scale bars, $100 \mu \mathrm{m}$. H Cell invasion of CHL-1 and SK-MEL-2 cells overexpressing RP11-705C15.3 or control were determined by the transwell invasion assay. Scale bars, $100 \mu \mathrm{m}$. Data are presented as mean \pm SD based on three independent experiments. $* p<0.05, \quad * * p<0.01, \quad * * * p<0.001$ by two-tailed unpaired $t$ test. with the tumors formed by control cells, which was also abolished by concurrent miR-145-5p overexpression (Fig. 6D). To elucidate the roles of RP11-705C15.3/miR-145-5p modulatory axis in melanoma metastasis in vivo, 

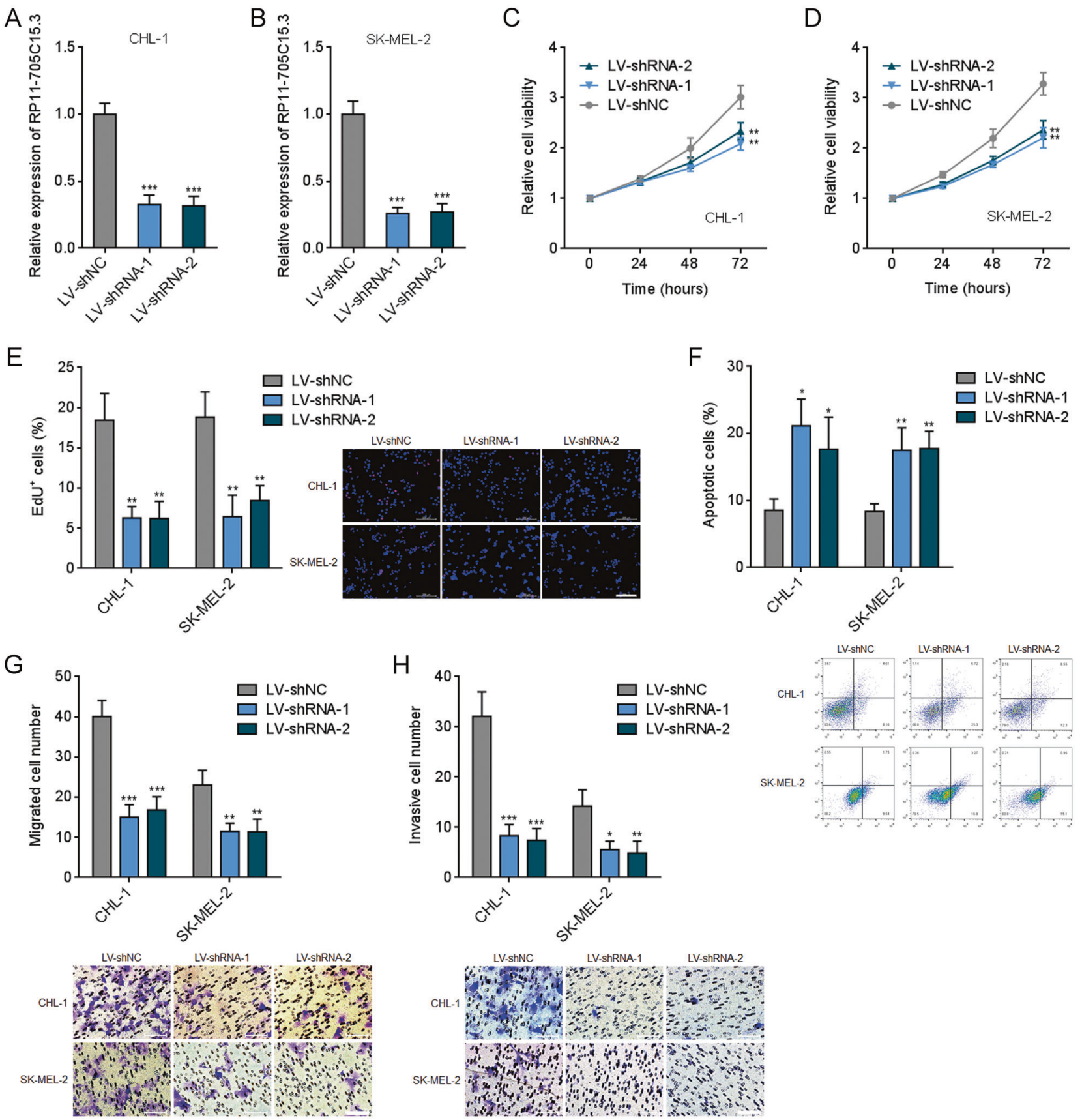

Fig. 4 RP11-705C15.3 silencing inhibited melanoma cell proliferation, induced apoptosis, and inhibited migration and invasion. A RP11-705C15.3 expression in CHL-1 cells silencing RP11-705C15.3 or control was measured by real-time PCR. B RP11$705 \mathrm{C} 15.3$ expression in SK-MEL-2 cells silencing RP11-705C15.3 or control was measured by real-time PCR. C Cell viabilities of CHL-1 cells silencing RP11-705C15.3 or control were determined by the Glo cell viability assay. D Cell viabilities of SK-MEL-2 cells silencing RP11-705C15.3 or control were determined by the Glo cell viability assay. E Cell proliferation of CHL-1 and SK-MEL-2 cells silencing RP11-705C15.3 or control were determined by the EdU incorporation

RP11-705C15.3 overexpressed CHL-1 cells with or without miR-145-5p overexpression were intrasplenically injected to construct liver metastasis model. As shown in Fig. 6E, assay. The red color indicates EdU-positive nuclei. Scale bars, $200 \mu \mathrm{m}$. F Cell apoptosis of CHL-1 and SK-MEL-2 cells silencing RP11$705 \mathrm{C} 15.3$ or control were determined by FITC-Annexin V/PI staining followed by flow cytometric analysis. G Cell migration of CHL-1 and SK-MEL-2 cells silencing RP11-705C15.3 or control were determined by the transwell migration assay. Scale bars, $100 \mu \mathrm{m}$. H Cell invasion of CHL-1 and SK-MEL-2 cells silencing RP11-705C15.3 or control were determined by the transwell invasion assay. Scale bars, $100 \mu \mathrm{m}$. Data are presented as mean \pm SD based on three independent experiments. $* p<0.05, * * p<0.01, * * * p<0.001$ by one-way ANOVA followed by Dunnett's multiple comparisons test.

RP11-705C15.3 overexpressed CHL-1 cells formed more liver metastases compared with control CHL-1 cells, which was abolished by concurrent miR-145-5p overexpression. 

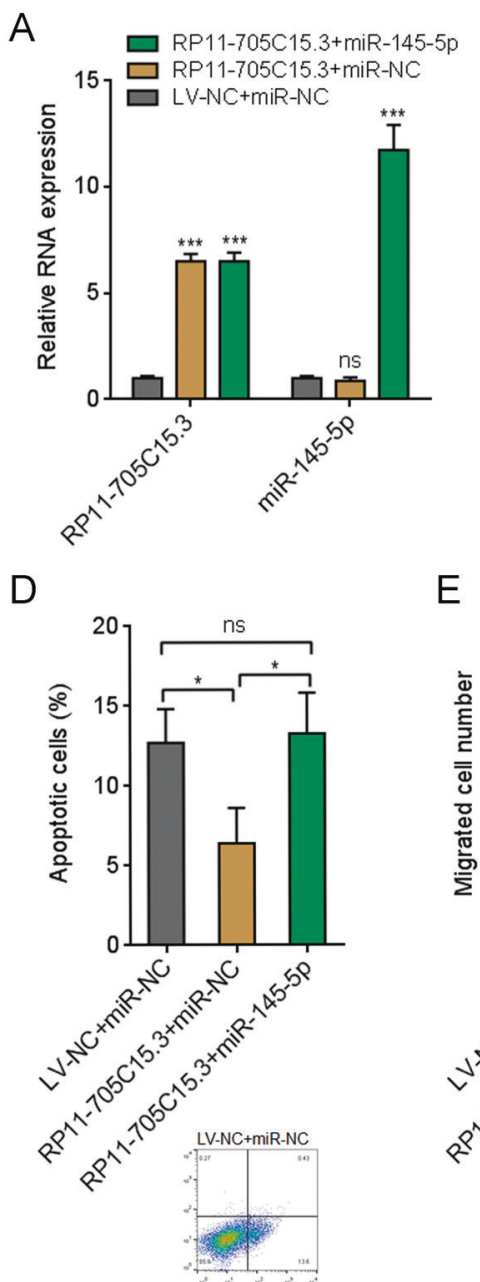

RP11-705C15.3+miR-NC RP11-705C15.3+miR-145-5

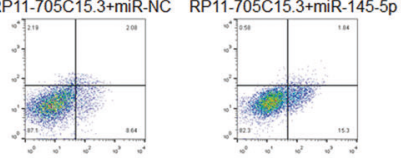

E
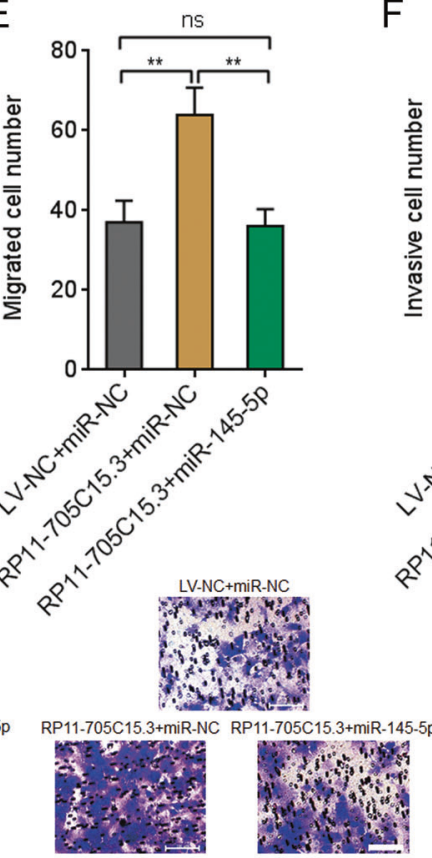

B

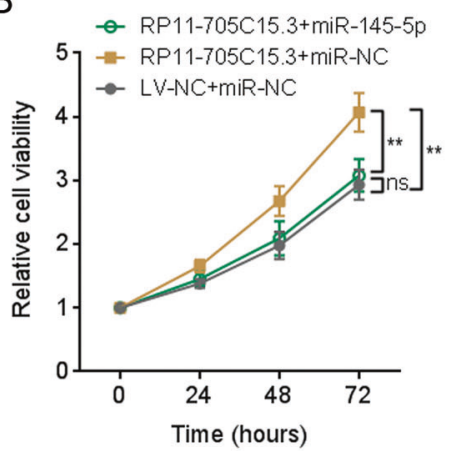

C
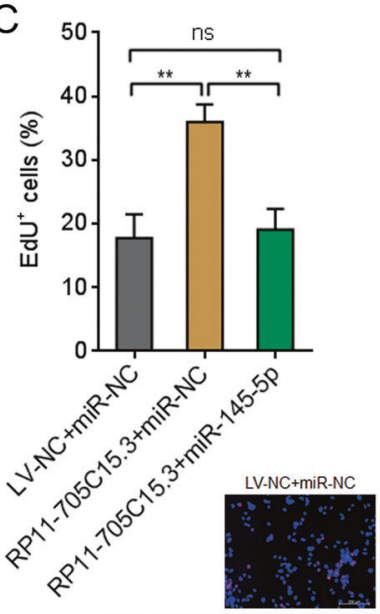

$\mathrm{F}$
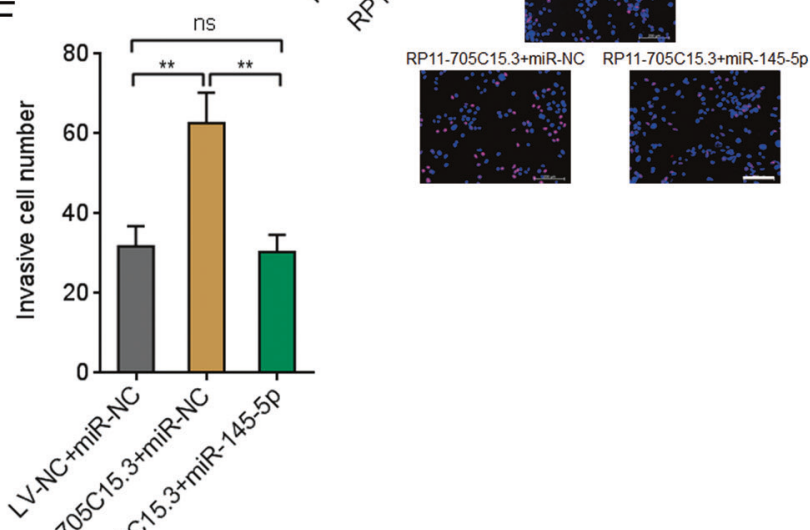

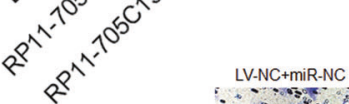
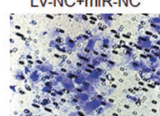

RP11-705C15.3+miR-NC RP11-705C15.3+miR-145-5p

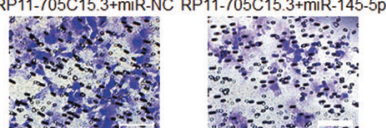

Fig. 5 Overexpression of miR-145-5p reversed the oncogenic roles of RP11-705C15.3 in melanoma. A RP11-705C15.3 and miR-145-5p expression levels in CHL-1 cells overexpressing RP11-705C15.3 and miR-145-5p were measured by real-time PCR. B Cell viabilities of CHL-1 cells overexpressing RP11-705C15.3 and miR-145-5p were determined by the Glo cell viability assay. C Cell proliferation of CHL-1 cells overexpressing RP11-705C15.3 and miR-145-5p were determined by the EdU incorporation assay. The red color indicates EdU-positive nuclei. Scale bars, $200 \mu \mathrm{m}$. D Cell apoptosis of CHL-1 cells overexpressing RP11-705C15.3 and miR-145-5p were

RP11-705C15.3 overexpressed CHL-1 cells with or without miR-145-5p overexpression were injected into the tail veins of nude mice to construct lung metastasis model. As shown in Fig. 6F, RP11-705C15.3 overexpressed CHL-1 cells formed more lung metastases compared with control CHL-1 cells, which was abolished by concurrent miR-145-5p overexpression. Thus, these findings suggested that RP11$705 \mathrm{C} 15.3$ promoted melanoma growth and metastasis in vivo in a miR-145-5p dependent manner. determined by FITC-Annexin V/PI staining followed by flow cytometric analysis. E Cell migration of CHL-1 cells overexpressing RP11-705C15.3 and miR-145-5p were determined by the transwell migration assay. Scale bars, $100 \mu \mathrm{m}$. F Cell invasion of CHL-1 cells overexpressing RP11-705C15.3 and miR-145-5p were determined by the transwell invasion assay. Scale bars, $100 \mu \mathrm{m}$. Data are presented as mean $\pm \mathrm{SD}$ based on three independent experiments. $* p<0.05$, $* * p<$ $0.01, * * * p<0.001, \mathrm{~ns}$, not significant, by one-way ANOVA followed by Tukey's multiple comparisons test.

\section{RP11-705C15.3 was correlated with poor clinical factors and inferior prognosis of melanoma}

To investigate the clinical significances of RP11-705C15.3 in melanoma, we first measured RP11-705C15.3 expression in 36 benign nevi and 68 melanoma tissues using real-time PCR. The results revealed that RP11-705C15.3 was significantly increased in melanoma tissues compared with benign nevi (Fig. 7A). Correlation analyses between RP11-705C15.3 

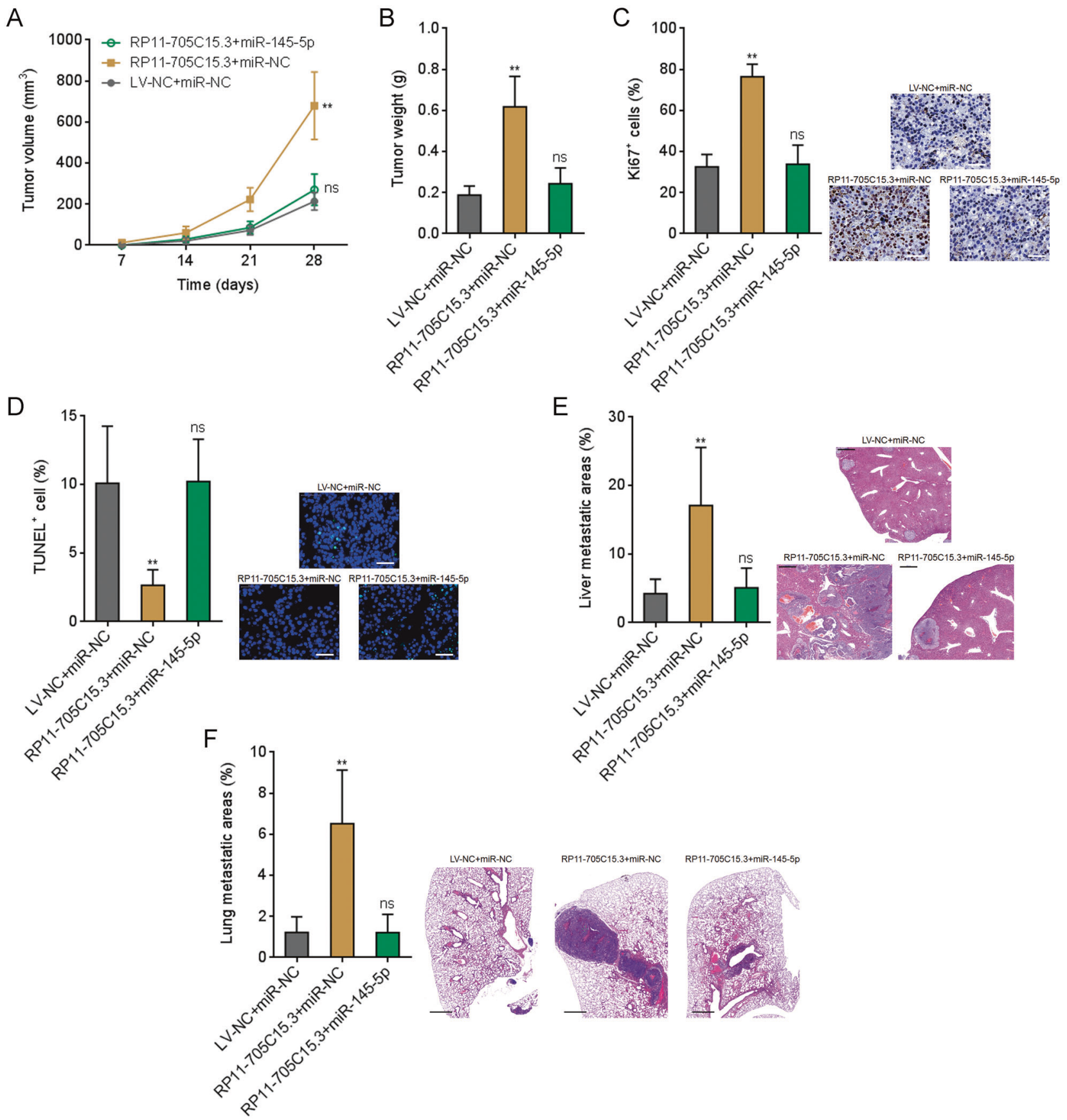

Fig. 6 RP11-705C15.3 promoted melanoma growth and metastasis in a miR-145-5p dependent manner. A-D CHL-1 cells overexpressing RP11-705C15.3 and miR-145-5p and control cells were subcutaneously inoculated into nude mice. Tumor volumes were measured every 7 days (A). The tumors were excised and weighed at the 28th day after inoculation (B). The tumors were used to perform Ki67 IHC staining to indicate in vivo proliferation (c). Scale bars, $50 \mu \mathrm{m}$. TUNEL staining was performed in these tumors to indicate cell apoptosis (D). Scale bars, $50 \mu \mathrm{m}$. E CHL-1 cells overexpressing RP11-

expression levels and clinicopathological characters revealed that increased expression levels of RP11-705C15.3 were correlated with thickness, ulceration, and metastasis (Fig. 7B-D). Moreover, Kaplan-Meier survival analysis revealed

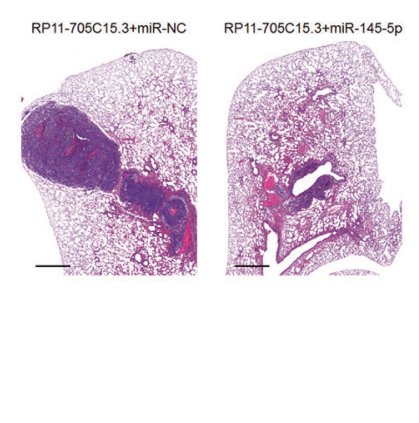

705C15.3 and miR-145-5p and control cells were inoculated into spleen of nude mice to construct liver metastasis model. The livers were excised and used to perform H\&E staining. Scale bars, $500 \mu \mathrm{m}$. F CHL-1 cells overexpressing RP11-705C15.3 and miR-145-5p and control cells were inoculated into tail vein of nude mice to construct lung metastasis model. The lungs were excised and used to perform H\&E staining. Scale bars, $1000 \mu \mathrm{m}$. Data are presented as mean \pm SD. $n=6$ mice in each group. $* * p<0.01$, ns, not significant, by Kruskal-Wallis test followed by Dunn's multiple comparisons test.

that increased expression levels of RP11-705C15.3 were correlated with inferior overall survival of melanoma patients (Fig. 7E). Thus, these findings suggested that RP11-705C15.3 was increased in melanoma and high expression of RP11- 
A
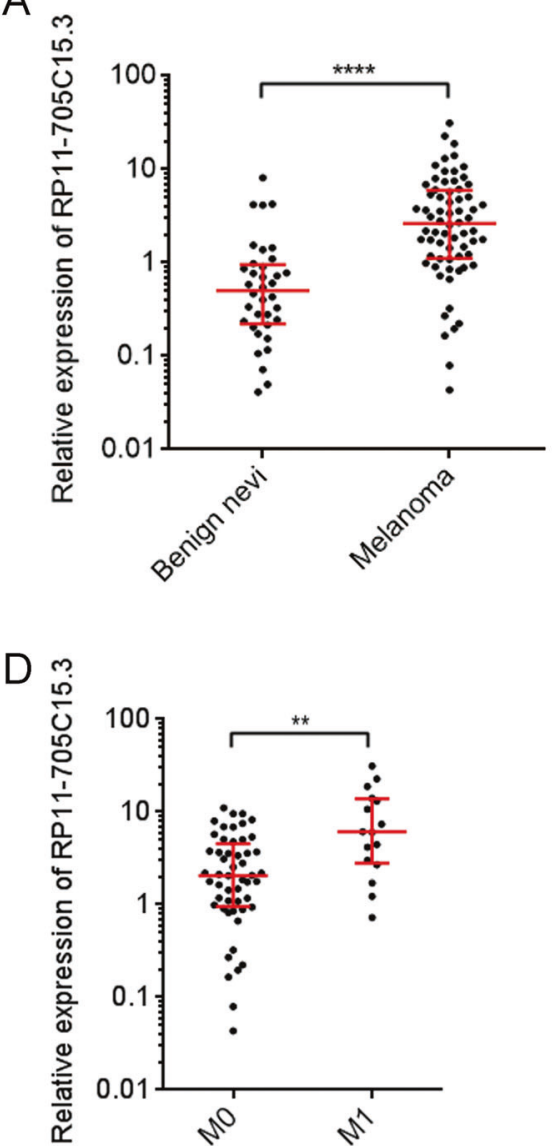

B

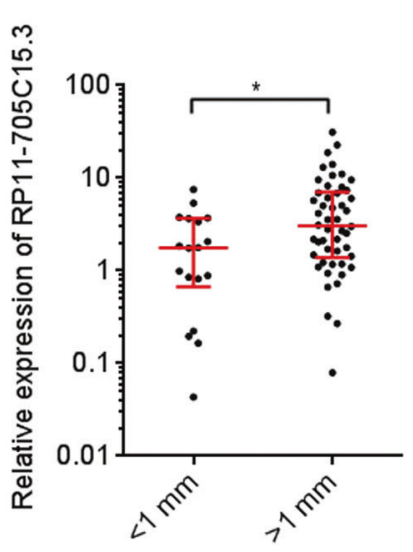

$E$

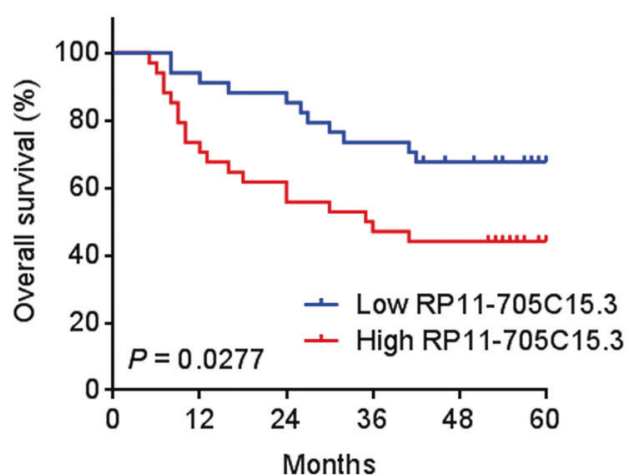

Fig. 7 RP11-705C15.3 was increased and correlated with poor clinical factors and prognosis of melanoma. A RP11-705C15.3 expression in 36 benign nevi and 68 cutaneous melanoma tissues was measured by real-time PCR. B RP11-705C15.3 expression in 18 melanoma tissues with thickness $<1 \mathrm{~mm}$ and 50 melanoma tissues with thickness $>1 \mathrm{~mm}$. C RP11-705C15.3 expression in 25 melanoma tissues without ulceration and 43 melanoma tissues with ulceration.

705C15.3 was correlated with poor clinical factors and inferior overall survival in melanoma.

\section{Discussion}

The gene encoding RP11-705C15.3 is located at chromosome 12p13.31 and has two exons. RP11-705C15.3 has $2508 \mathrm{nt}$ in length. The knowledge of RP11-705C15.3 in diseases is lacking. In this study, we first identified RP11-705C15.3 as a melanoma-related lncRNA. Our findings revealed that RP11-705C15.3 is upregulated in melanoma. Increased expression of RP11-705C15.3 is positively correlated with thickness, ulceration, metastasis, and inferior prognosis of melanomas. Thus, RP11$705 \mathrm{C} 15.3$ is suggested as a potential prognostic marker for melanoma.
C

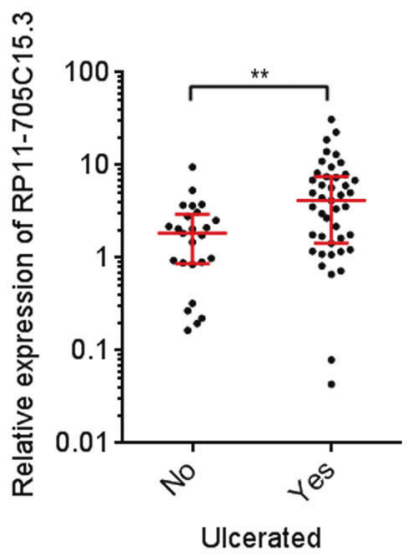

D RP11-705C15.3 expression in 52 melanoma tissues without distant metastasis and 16 primary melanoma tissues with distant metastasis. For $\mathbf{A}-\mathbf{D}$, data are presented as median with interquartile range. ${ }^{*} p<$ $0.05, \quad * * p<0.01, \quad * * * * p<0.0001$ by Mann-Whitney test. E Kaplan-Meier survival curves of these 68 melanomas stratified by RP11-705C15.3 expression (low 50\% $[n=34]$ versus high 50\% $[n=$ 34]). $p=0.0277$ by log-rank test.

Gain and loss-of-function assays revealed that RP11705C15.3 promotes melanoma cell proliferation, represses cell apoptosis, and promotes cell migration and invasion in vitro. Furthermore, RP11-705C15.3 promotes melanoma growth and metastasis in vivo. Thus, these functional experiments revealed that RP11-705C15.3 has oncogenic roles in melanoma and repressing RP11-705C15.3 could inhibit melanoma progression. These findings suggest RP11$705 \mathrm{C} 15.3$ as a potential therapeutic target for melanoma.

The mechanisms of actin of IncRNAs are complex and different [31]. One of the major mechanisms of IncRNAs is to interact with proteins, change the post-translational modification, stability, and/or location, and thus lastly modulate the functions of the interacted proteins [32-34]. lncRNA SAMMSON is reported to interact with p32 and further increase mitochondrial targeting of p32 and the pro-oncogenic function of p32 [32]. IncRNA SLNCR1 is 
reported to bind androgen receptor and Brn3a, which further upregulates MMP9 [33]. CASC15 is reported to bind and recruit EZH2 to the promoter region of $P D C D 4$, and therefore silence PDCD4 expression [34]. Another major mechanism of lncRNAs is to directly interact with miRNAs and relieve the repressive roles of miRNAs on their targets. These lncRNAs are classed as ceRNAs [35-37]. lncRNA NEAT1 is reported to bind miR-495-3p and upregulate E2F3, a target of miR-495-3p [35]. IncRNA LINC00459 is revealed to bind miR-218 and elevate DKK3, a target of miR-218 [36]. lncRNA-ATB is reported to bind miR-200s and upregulate ZEB1 and ZEB2, targets of miR-200s [37].

In this study, our findings revealed that RP11$705 \mathrm{C} 15.3$ is majorly localized in cytoplasm. Cytoplasmic RP11-705C15.3 specifically binds to mature miR145-5p and elevates NRAS, a critical target of miR-145$5 p$ in cancers $[38,39]$. Several reports, including our own, all demonstrate the critical tumor-suppressive roles of miR-145-5p [28, 40, 41]. Consistently, in this study, our data revealed that via competitively binding miR-145-5p, RP11-705C15.3 exerts oncogenic roles in melanoma. miR-145-5p overexpression reversed the roles of RP11$705 \mathrm{C} 15.3$ in promoting cell proliferation, inhibiting apoptosis, and promoting migration and invasion. Moreover, in vivo xenografts assays revealed that miR-145-5p overexpression reversed the roles of RP11-705C15.3 in promoting melanoma tumor growth and metastasis. These findings supported miR-145-5p as an important downstream mediator of RP11-705C15.3 in melanoma. Via competitively binding miR-145-5p and elevating NRAS, RP11-705C15.3 activates RAF/MAPK signaling. MAPK signaling has been reported to be frequently activated in melanoma [42]. Apart from NRAS, other molecules were also identified as miR-145-5p targets, such as RHBDD1, FSCN1, and Sox9 [40, 41, 43]. The effects of RP11$705 \mathrm{C} 15.3$ on other miR-145-5p targets need further investigation.

In summary, we identified RP11-705C15.3 as a prognosis-related and oncogenic lncRNA in melanoma through competitively binding miR-145-5p and activating NRAS/MAPK signaling axis. These findings provide new insights about the molecular events underlying melanoma progression and potential molecule targets for melanoma prognosis and therapy.

Acknowledgements This work was supported by the National Natural Science Foundation of China (81971854).

\section{Compliance with ethical standards}

Conflict of interest The authors declare that they have no conflict of interest.
Publisher's note Springer Nature remains neutral with regard to jurisdictional claims in published maps and institutional affiliations.

Open Access This article is licensed under a Creative Commons Attribution 4.0 International License, which permits use, sharing, adaptation, distribution and reproduction in any medium or format, as long as you give appropriate credit to the original author(s) and the source, provide a link to the Creative Commons license, and indicate if changes were made. The images or other third party material in this article are included in the article's Creative Commons license, unless indicated otherwise in a credit line to the material. If material is not included in the article's Creative Commons license and your intended use is not permitted by statutory regulation or exceeds the permitted use, you will need to obtain permission directly from the copyright holder. To view a copy of this license, visit http://creativecommons. org/licenses/by/4.0/.

\section{References}

1. Orgaz JL, Crosas-Molist E, Sadok A, Perdrix-Rosell A, Maiques $\mathrm{O}$, Rodriguez-Hernandez I. et al. Myosin II reactivation and cytoskeletal remodeling as a hallmark and a vulnerability in melanoma therapy resistance. Cancer Cell. 2020;37:85-103.e9.

2. Siegel RL, Miller KD, Jemal A. Cancer statistics, 2020. CA Cancer J Clin. 2020;70:7-30.

3. Bray F, Ferlay J, Soerjomataram I, Siegel RL, Torre LA, Jemal A. Global cancer statistics 2018: GLOBOCAN estimates of incidence and mortality worldwide for 36 cancers in 185 countries. CA Cancer J Clin. 2018;68:394-424.

4. Gide TN, Quek C, Menzies AM, Tasker AT, Shang P, Holst J. et al. Distinct immune cell populations define response to AntiPD-1 monotherapy and anti-PD-1/anti-CTLA-4 combined therapy. Cancer Cell. 2019;35:238-55.e6.

5. Rozeman EA, Dekker TJA, Haanen J, Blank CU. Advanced melanoma: current treatment options, biomarkers, and future perspectives. Am J Clin Dermatol. 2018;19:303-17.

6. Iyer MK, Niknafs YS, Malik R, Singhal U, Sahu A, Hosono Y, et al. The landscape of long noncoding RNAs in the human transcriptome. Nat Genet. 2015;47:199-208.

7. Ponting CP, Oliver PL, Reik W. Evolution and functions of long noncoding RNAs. Cell. 2009;136:629-41.

8. Esposito R, Bosch N, Lanzos A, Polidori T, Pulido-Quetglas C, Johnson R. Hacking the cancer genome: profiling therapeutically actionable long non-coding RNAs using CRISPR-Cas9 screening. Cancer Cell. 2019;35:545-57.

9. Li Y, Yan G, Zhang J, Chen W, Ding T, Yin Y, et al. LncRNA HOXA11-AS regulates calcium oxalate crystal-induced renal inflammation via miR-124-3p/MCP-1. J Cell Mol Med. 2020;24:238-49.

10. Khaitan D, Dinger ME, Mazar J, Crawford J, Smith MA, Mattick JS, et al. The melanoma-upregulated long noncoding RNA SPRY4-IT1 modulates apoptosis and invasion. Cancer Res. 2011;71:3852-62.

11. Wu CF, Tan GH, Ma CC, Li L. The non-coding RNA llme23 drives the malignant property of human melanoma cells. J Genet Genomics. 2013;40:179-88.

12. Sang B, Zhang YY, Guo ST, Kong LF, Cheng Q, Liu GZ, et al. Dual functions for OVAAL in initiation of RAF/MEK/ERK prosurvival signals and evasion of p27-mediated cellular senescence. Proc Natl Acad Sci USA. 2018;115:E11661-E70.

13. Hong $\mathrm{CH}$, Ho JC, Lee CH. Steroid receptor RNA activator, a long noncoding RNA, activates p38, facilitates epithelial-mesenchymal transformation, and mediates experimental melanoma metastasis. J Invest Dermatol. 2020;140:1355-63.e1. 
14. Luan W, Ding Y, Yuan H, Ma S, Ruan H, Wang J, et al. Long non-coding RNA LINC00520 promotes the proliferation and metastasis of malignant melanoma by inducing the miR-125b-5p/ EIF5A2 axis. J Exp Clin Cancer Res. 2020;39:96.

15. Chen X, Gao G, Liu S, Yu L, Yan D, Yao X, et al. Long noncoding RNA PVT1 as a novel diagnostic biomarker and therapeutic target for melanoma. BioMed Res Int. 2017;2017:7038579.

16. Chen X, Liu S, Zhao X, Ma X, Gao G, Yu L, et al. Long noncoding RNA ILF3-AS1 promotes cell proliferation, migration, and invasion via negatively regulating miR-200b/a/429 in melanoma. Biosci Rep. 2017;37:BSR20171031.

17. Chen X, Dong H, Liu S, Yu L, Yan D, Yao X, et al. Long noncoding RNA MHENCR promotes melanoma progression via regulating miR-425/489-mediated PI3K-Akt pathway. Am J Transl Res. 2017;9:90-102.

18. Zhu XT, Yuan JH, Zhu TT, Li YY, Cheng XY. Long noncoding RNA glypican 3 (GPC3) antisense transcript 1 promotes hepatocellular carcinoma progression via epigenetically activating GPC3. FEBS J. 2016;283:3739-54.

19. Li JK, Chen C, Liu JY, Shi JZ, Liu SP, Liu B, et al. Long noncoding RNA MRCCAT1 promotes metastasis of clear cell renal cell carcinoma via inhibiting NPR3 and activating p38-MAPK signaling. Mol Cancer. 2017;16:111.

20. Mo S, Zhang L, Dai W, Han L, Wang R, Xiang W, et al. Antisense lncRNA LDLRAD4-AS1 promotes metastasis by decreasing the expression of LDLRAD4 and predicts a poor prognosis in colorectal cancer. Cell Death Dis. 2020;11:155.

21. Cesana M, Cacchiarelli D, Legnini I, Santini T, Sthandier O, Chinappi $\mathrm{M}$, et al. A long noncoding RNA controls muscle differentiation by functioning as a competing endogenous RNA. Cell. 2011;147:358-69.

22. Salmena L, Poliseno L, Tay Y, Kats L, Pandolfi PP. A ceRNA hypothesis: the Rosetta Stone of a hidden RNA language? Cell. 2011;146:353-8.

23. Jiang Q, Isquith J, Zipeto MA, Diep RH, Pham J, Delos Santos N. et al. Hyper-editing of cell-cycle regulatory and tumor suppressor RNA promotes malignant progenitor propagation. Cancer Cell. 2019;35:81-94.e7.

24. Yuan JH, Yang F, Chen BF, Lu Z, Huo XS, Zhou WP, et al. The histone deacetylase 4/SP1/microrna-200a regulatory network contributes to aberrant histone acetylation in hepatocellular carcinoma. Hepatology. 2011;54:2025-35.

25. Bartel DP. MicroRNAs: genomics, biogenesis, mechanism, and function. Cell. 2004;116:281-97.

26. Li MM, Liu XH, Zhao YC, Ma XY, Zhou YC, Zhao YX, et al. Long noncoding RNA KCNQ1OT1 promotes apoptosis in neuroblastoma cells by regulating miR-296-5p/Bax axis. FEBS J. 2020;287:561-77.

27. Cheng Y, Shen P. miR-335 acts as a tumor suppressor and enhances ionizing radiation-induced tumor regression by targeting ROCK1. Front Oncol. 2020;10:278.

28. Liu S, Gao G, Yan D, Chen X, Yao X, Guo S, et al. Effects of miR-145-5p through NRAS on the cell proliferation, apoptosis, migration, and invasion in melanoma by inhibiting MAPK and PI3K/AKT pathways. Cancer Med. 2017;6:819-33.

29. van den Berg A, Mols J, Han J. RISC-target interaction: cleavage and translational suppression. Biochim Biophys Acta. 2008; 1779:668-77.

30. Li JH, Liu S, Zhou H, Qu LH, Yang JH. starBase v2.0: decoding miRNA-ceRNA, miRNA-ncRNA and protein-RNA interaction networks from large-scale CLIP-Seq data. Nucleic Acids Res. 2014;42:D92-7.

31. Yuan JH, Liu XN, Wang TT, Pan W, Tao QF, Zhou WP, et al. The MBNL3 splicing factor promotes hepatocellular carcinoma by increasing PXN expression through the alternative splicing of lncRNA-PXN-AS1. Nat Cell Biol. 2017;19:820-32.

32. Leucci E, Vendramin R, Spinazzi M, Laurette P, Fiers M, Wouters $\mathrm{J}$, et al. Melanoma addiction to the long non-coding RNA SAMMSON. Nature. 2016;531:518-22.

33. Schmidt K, Joyce CE, Buquicchio F, Brown A, Ritz J, Distel RJ, et al. The lncRNA SLNCR1 mediates melanoma invasion through a conserved SRA1-like region. Cell Rep. 2016;15:2025-37.

34. Yin Y, Zhao B, Li D, Yin G. Long non-coding RNA CASC15 promotes melanoma progression by epigenetically regulating PDCD4.Cell Biosci. 2018;8:42.

35. Xia Y, Zhou Y, Han H, Li P, Wei W, Lin N. lncRNA NEAT1 facilitates melanoma cell proliferation, migration, and invasion via regulating miR-495-3p and E2F3. J Cell Physiol. 2019;234:19592-601.

36. Yang Y, Xu W, Zheng Z, Cao Z. LINC00459 sponging miR-218 to elevate DKK3 inhibits proliferation and invasion in melanoma. Sci Rep. 2019;9:19139.

37. Yuan JH, Yang F, Wang F, Ma JZ, Guo YJ, Tao QF, et al. A long noncoding RNA activated by TGF-beta promotes the invasionmetastasis cascade in hepatocellular carcinoma. Cancer Cell. 2014;25:666-81.

38. Yin Y, Yan ZP, Lu NN, Xu Q, He J, Qian X, et al. Downregulation of miR-145 associated with cancer progression and VEGF transcriptional activation by targeting N-RAS and IRS1. Biochim Biophys Acta. 2013;1829:239-47.

39. Zou C, Xu Q, Mao F, Li D, Bian C, Liu LZ, et al. MiR-145 inhibits tumor angiogenesis and growth by N-RAS and VEGF. Cell Cycle. 2012;11:2137-45.

40. Niu Y, Zhang J, Tong Y, Li J, Liu B. miR-145-5p restrained cell growth, invasion, migration and tumorigenesis via modulating RHBDD1 in colorectal cancer via the EGFR-associated signaling pathway. Int J Biochem Cell Biol. 2019;117:105641.

41. Fuse M, Nohata N, Kojima S, Sakamoto S, Chiyomaru T, Kawakami K, et al. Restoration of miR-145 expression suppresses cell proliferation, migration and invasion in prostate cancer by targeting FSCN1. Int J Oncol. 2011;38:1093-101.

42. Romano E, Schwartz GK, Chapman PB, Wolchock JD, Carvajal RD. Treatment implications of the emerging molecular classification system for melanoma. Lancet Oncol. 2011;12:913-22.

43. Rani SB, Rathod SS, Karthik S, Kaur N, Muzumdar D, Shiras AS. MiR-145 functions as a tumor-suppressive RNA by targeting Sox9 and adducin 3 in human glioma cells. Neuro Oncol. 2013;15:1302-16. 